\title{
Probability, Indeterminism and Biological Processes
}

Charlotte Werndl

Lecturer, Department of Philosophy, Logic and Scientific Method

London School of Economics and Political Science

Houghton Street

London WC2A 2AE, UK

c.s.werndl@lse.ac.uk

This article is forthcoming in:

D. Dieks, J.G. Wenceslao, S. Hartmann, M. Stoeltzner, and M. Weber (eds), Probabilities, Laws and Structures. The Philosophy of Science in a European Perspective, Volume 3. Springer.

\section{Introduction}

Probability and indeterminism have always been core philosophical themes. Biology provides an interesting case study to explore these themes. First, biology is teeming with probabilities, and so a crucial question in the foundations of biology is how to understand these probabilities. Second, philosophers want to know whether the processes investigated by one of the major sciences - biology - are indeterministic.

This paper aims to contribute to understanding probability and indeterminism in biology. More specifically, Section 2 will provide the background for the paper. It will be argued that an omniscient being would not need the probabilities of evolutionary theory to make predictions about biological processes. However, despite this, one can still be a realist about evolutionary theory, and then the probabilities in evolutionary theory refer to real features of the world. This prompts the question of how to interpret biological probabilities which correspond to real features of the world but are in principle dispensable for predictive purposes. Section 3 will suggest three possible interpretations of such probabilities. The first interpretation is a propensity interpretation of kinds of systems. It will be argued that, contra Sober ${ }^{1}$, backward probabilities in biology do not present a problem for the propensity interpretation. The second interpretation is the frequency interpretation, and it will be argued that Millstein's ${ }^{2}$ objection against this interpretation in evolutionary theory is beside the point. Finally, I will suggest Humean chances are a new interpretation of probability in evolutionary theory. Section 4 discusses Sansom's ${ }^{3}$ argument that biological processes are indeterministic because probabilities in evolutionary theory refer to real features of the world. It will be argued that Sansom's argument is not conclusive, and that the question whether biological processes are deterministic or indeterministic is still with us.

\footnotetext{
${ }^{1}$ Elliott Sober, "Evolutionary Theory and the Reality of Macro Probabilities", Philosophy of Science Presidential Address 2004.

2 Roberta L. Millstein, "Interpretations of Probability in Evolutionary Theory", in: Philosophy of Science 70, 4, 2003, pp.1317-1328.

${ }^{3}$ Robert Sansom, "Why Evolution is Really Indeterministic", in: Synthese 136, 2, 2003, pp.263-280.
} 


\section{Realism, Indeterminism and Omniscient Beings}

This section provides the background for the paper. First, the notions of realism, instrumentalism, determinism and indeterminism will be introduced. Then it will be explained that an omniscient being would not need the probabilities of evolutionary theory to make predictions about biological processes. It is argued that, despite this, one can still be a realist about evolutionary theory.

(Scientific) realism about a theory $T$ is the idea that $T$ corresponds to the world, i.e., $T$ gives at least an approximately true description of the real-world processes falling under its scope. Instrumentalism relative to a theory $T$ as understood in this paper is the negation of realism. Hence an instrumentalist about a theory $T$ denies that $T$ corresponds to the world. For what follows a definition of determinism for theories as well as for real-world processes is needed. A theory $T$ is deterministic if and only if a state description of a system is always followed by the same history of transitions of state descriptions. A theory $T$ is indeterministic if and only if it is not deterministic. A process is deterministic (concerning a specific set of kinds) if and only if a given state of a kind is always followed by the same history of transitions of states of kinds. ${ }^{4} \mathrm{~A}$ process is indeterministic (concerning a specific set of kinds) if and only if it is not deterministic. $^{5}$

Probabilities are of utmost importance in evolutionary theory, and the probabilistic character of evolutionary theory is widely accepted. ${ }^{6}$ An example is the concept of fitness of an organism in an environment (see Section 3 for other examples of probabilities in evolutionary theory). Since one wants to allow that in unusual circumstances less fit organisms have more offspring than fitter ones, fitness of an organism $^{7}$ is captured by means of the probability to have a certain level of reproductive success. ${ }^{8}$

An omniscient being would not need the probabilities of evolutionary theory to make predictions about biological processes. The next two paragraphs will explain why this is so. In essence, this is a consequence of the fact that evolutionary theory ignores certain details and factors. For example, evolutionary theory does not include detailed models of flashes of lightning (because which organisms will struck by lightning is random - i.e., not related to their actual traits). Another example is that the exact location at each point of time of a chimpanzee in a forest does not appear as a variable in evolutionary theory (because this location is not correlated to reproductive success). Now consider models which correctly describe biological processes in all their details at the level of macrophysics. These macro-physical models will be very different from models in evolutionary theory because the former include details and factors which are ignored by the latter. For example, such macro-physical models

\footnotetext{
${ }^{4}$ Jeremy Butterfield, "Determinism and Indeterminism", in: Routledge Encyclopaedia of Philosophy Online 2005. John Earman, A Primer on Determinism. Dotrecht: D. Reidel Publishing 1986.

${ }^{5}$ What I call "determinism" of theories and processes is also sometimes called "future determinism". This is to highlight that it is not required that any state is also always preceded by the same history of transitions of states (see Earman, Ibid., pp.13-14).

${ }^{6}$ Sansom, Ibid., pp.268-269.

${ }^{7}$ Robert N. Brandon, Adaptation and Environment. Princeton: Princeton University Press 1990, p.15.

${ }^{8}$ How to define or measure fitness exactly turns out to be tricky. For an organism in a specific environment it is not enough to consider the expected value of offspring number, sometimes also the variance and other measures need to be taken into consideration (cf. Brandon "Adaptation and Environment", loc. cit., p.20).
} 
include a description of flashes of lightening and they include variables for the exact location of chimpanzees.

Are these macro-physical models of biological processes deterministic or indeterministic? This is a matter of debate. Rosenberg ${ }^{9}$ argues that they are deterministic. Abrams ${ }^{10}$ and Graves et al. ${ }^{11}$ claim that these models are "nearly deterministic". What is meant by this is that they are indeterministic because quantum mechanical probabilities can percolate up and quantum mechanics is indeterministic. ${ }^{12}$ However, because macro-physical objects consist of many particles, the probabilities at the macro-level are very close to zero or one. Others such as Millstein ${ }^{13}$ and Weber ${ }^{14}$ argue that we do not know enough about the role of quantum events at the macroscopic level and hence should remain agnostic: these models could be deterministic or indeterministic with probabilities very close to zero or one. The upshot is that even if there are nontrivial probabilities for macro-physical models of biological processes, they are different from those probabilities figuring in evolutionary theory. Consequently, evolutionary theory appeals to probabilities which at least partly arise from ignoring certain details and factors. Hence an omniscient being would not have to rely on the probabilities of evolutionary theory to make predictions about biological processes. If the world at the macro-physical level is deterministic, an omniscient being could appeal to a deterministic theory to predict biological processes. If the world at the macro-physical level is indeterministic, the omniscient being could appeal to a very different indeterministic theory (with probabilities close to zero and one) to predict biological processes.

Does this have any implications about whether one should be a realist or instrumentalist about evolutionary theory? Rosenberg thinks so. Because an omniscient being would not need evolutionary theory, he argues that "This makes our actual theory of natural selection more of a useful instrument than a set of

\footnotetext{
${ }^{9}$ Alexander Rosenberg, Instrumental Biology or the Disunity of Science. Chicago: The University of Chicago Press 1994.

${ }^{10}$ Marshall Abrams, "Fitness and Propensity’s Annulment?", in: Biology and Philosophy 22, 1, 2007, pp.115-130.

${ }^{11}$ Leslie Graves, Barbara L. Horan and Alexander Rosenberg, "Is Indeterminism the Source of the Probabilistic Character of Evolutionary Theory?", in: Philosophy of Science 66, 1999, 1, pp.140-157. See also Alexander Rosenberg, "Discussion Note: Indeterminism, Probability, and Randomness in Evolutionary Theory", in: Philosophy of Science 68, 4, 2001, pp.536-544.

12 These positions and generally philosophers of biology take it to be uncontroversial that quantum theory is indeterministic (see Abrams "Fitness and Propensity's Annulment?", loc. cit., pp.119-121; Graves et al, Ibid., pp.144-145; Rosenberg "Discussion Note: Indeterminism, Probability, and Randomness in Evolutionary Theory", loc. cit., pp.537-538; Sansom Ibid., p.267). However, this is questionable. As generally agreed in philosophy of physics, there are coherent deterministic interpretations of quantum theory and "the alleged indeterminism of quantum theory is very controversial: it enters, if at all, only in quantum theory's account of measurement processes, an account which remains the most controversial part of the theory" (Butterfield, Ibid.). Similarly, it is often simply assumed that macrophysics is deterministic (e.g. Graves et al., Ibid., p.145; Rosenberg "Discussion Note: Indeterminism, Probability, and Randomness in Evolutionary Theory", loc. cit., p.537). Yet, research in philosophy of physics has shown that it is unclear whether macrophysics is deterministic (see Earman, Ibid., Chapter III). These assumptions are questionable, but they will not matter for what follows.

${ }^{13}$ Roberta L. Millstein, "Is the Evolutionary Process Deterministic or Indeterministic? An Argument for Agnosticism", Presented at the Biennial Meeting of the Philosophy of Science Association Vancouver, Canada, 2000.

${ }^{14}$ Marcel Weber, "Indeterminism in Neurobiology", in: Philosophy of Science (Proceedings) 71, 2005, 663-674.
} 
propositions about the world independent of our beliefs about it."15 So Rosenberg argues that because an omniscient being would not need evolutionary theory, this implies instrumentalism about evolutionary theory.

Weber ${ }^{16}$ disagrees with Rosenberg. He points out that:

"A theory may be dispensable in the sense that an omniscient being would be able to understand the phenomena in question at a deeper level, but it is still possible that this theory correctly represents some aspects of reality. To put it differently, a theory may be indispensable merely for pragmatic reasons i.e., for reasons which have to do with our cognitive abilities, but still be open to a realist interpretation. The fact that a theory falls short of giving us a complete account of some complex causal processes does not imply that this theory has no representational content whatsoever. A scientific realist is not committed to the thesis that even our best scientific theories provide complete descriptions of reality." $" 17$

In my opinion, Rosenberg is in principle right that the dispensability of evolutionary theory for an omniscient being can lead to the rejection of realism about evolutionary theory. However, this is only the case when one endorses an extremely strong version of realism, viz. a realism which demands that theories should match reality to such a high degree that an omniscient being could not use another theory to predict the processes in question. Weber correctly points out that such a strong version of realism is hard to swallow. ${ }^{18}$ Hence one can be a realist about evolutionary theory even if an omniscient being would not have to rely on evolutionary theory to predict biological processes. To give an example, assume that Newtonian mechanics truly describes the world. Then, according to Rosenberg's argument, it would follow that one cannot be a realist about statistical mechanics. Yet, most physicists and philosophers contend that it is possible to be a realist about statistical mechanics: statistical mechanics correctly represents certain features of systems even if these systems can be described in more detail at the microscopic level by Newtonian mechanics. ${ }^{19}$

To conclude, one can still be a realist about evolutionary theory even it this theory is dispensable for an omniscient being for predictive purposes. Many biologists and philosophers of biology are realists in such a sense, and then the interesting question arises of how to interpret the probabilities figuring in evolutionary theory. Because of realism, these probabilities are ontic in the sense that they refer to real feature of the world. ${ }^{20}$ Yet, an omniscient being would not need these probabilities to make predictions (because an omniscient being could use a more fine-grained theory which is either deterministic or invokes probabilities different from evolutionary theory). So

\footnotetext{
${ }^{15}$ Rosenberg "Instrumental Biology or the Disunity of Science", loc. cit., p.83.

${ }^{16}$ Marcel Weber, "Determinism, Realism, and Probability in Evolutionary Theory", in: Philosophy of Science (Proceedings) 68, 2001, pp.213-224.

${ }^{17}$ Weber "Determinism, Realism, and Probability in Evolutionary Theory", loc. cit., p.217, original emphasis.

${ }^{18}$ For an example of a kind of scientific realism that does not demand that our best scientific theories provide complete descriptions of reality, see Kenneth C. Waters, "Tempered Realism About the Force of Selection", in: Philosophy of Science 58, 4, 1991, pp.553-573.

19 Roman Frigg, "A Field Guide to Recent Work on the Foundations of Statistical Mechanics", in: Dean Rickles (Ed.), The Ashgate Companion to Contemporary Philosophy of Physics. London: Ashgate 2008, pp.99-196.

${ }^{20}$ Hugh Mellor, Probability: A Philosophical Introduction. Cambridge: Cambridge University Press 2005. Mellor calls these probabilities "chances". I prefer the term "ontic" because some philosophers think that the term "chance" should only be used to refer to probabilities in an indeterministic world.
} 
the task is to find interpretations of ontic probabilities which could in principle be eliminated for predictive purposes.

\section{Interpretations of Ontic Probabilities in Evolutionary Theory}

This section will discuss three possible interpretations of ontic probabilities in evolutionary theory consistent with the claim that the probabilities are in principle dispensable for predictive purposes, namely a propensity interpretation of kinds of systems (Section 3.1), the frequency interpretation (Section 3.2) and Humean chances (Section 3.3). It is worth pointing out that also in several other contexts scientists and philosophers talk about ontic probabilities which are in principle dispensable for predictive purposes. Examples are setups where the world is supposed to be deterministic at a more fundamental level, such as the probabilities in statistical mechanics or the probabilities arising from coin tosses, roulette wheels and similar processes. $^{21}$

Millstein $^{22}$ already proposes two versions of the propensity account as possible interpretations of probability consistent with both determinism and indeterminism (hence these interpretations are consistent with the claim that probabilities are in principle dispensable for predictive purposes). The discussion of this paper differs in four respects. First, two interpretations are suggested which were not suggested by Millstein. In particular, I propose Humean chances as a possible interpretation of biological probabilities, and to the best of my knowledge, Humean chances have not previously been suggested as an interpretation of probabilities in evolutionary theory. Second, Sober's ${ }^{23}$ objection to the propensity interpretation based on backward probabilities in biology is examined and dismissed; this objection has not been discussed by Millstein. Third, as outlined below, I disagree with Millstein's argument against frequency interpretations in evolutionary theory. Fourth, Millstein ${ }^{24}$ proposes an interpretation based on Giere's single-case propensity interpretation. Single-case propensities provide an interpretation of probabilities that are not in principle dispensable for predictive purposes. ${ }^{25}$ Hence this interpretation cannot be applied to probabilities as they arise in evolutionary theory. Yet Giere ${ }^{26}$ suggests, and Millstein follows him in this, that from a pragmatic perspective his interpretation of probability can also be applied to probabilities that are in principle dispensable but behave like if there were not dispensable. However, if one makes this pragmatic move, one does not understand what probabilities are, and one cannot say that probabilities really exist. Consequently, I do not think that interpreting Giere's account pragmatically leads to a satisfying interpretation of probabilities which are in principle dispensable for predictive purposes.

\footnotetext{
${ }^{21}$ Frigg, Ibid.; Mellor, Ibid., p.55.

${ }^{22}$ Roberta L. Millstein, "Interpretations of Probability in Evolutionary Theory", in: Philosophy of Science 70, 4, 2003, pp,1317-1328.

${ }^{23}$ Sober, "Evolutionary Theory and the Reality of Macro Probabilities", loc. cit.

${ }^{24}$ Millstein "Interpretations of Probability in Evolutionary Theory”, loc. cit., pp.1322-1324.

${ }^{25}$ Ronald N. Giere, "Objective Single-Case Probabilities and the Foundations of Statistics", in: Patrick Suppes, Leon Henkin, Grigore Moisil and Athanase Joja (Eds.), Logic, Methodology, and the Philosophy of Science. North Holland: Amerikan Elsevier, 1973, pp.467-483.

${ }^{26}$ Giere "Objective Single-Case Probabilities and the Foundations of Statistics", loc. cit., p.481.
} 


\subsection{Propensity Interpretation}

The three interpretations of ontic probabilities will now be presented. The first interpretation is version of the propensity interpretation, namely what Millstein calls a "propensity interpretation that views propensities as adhering to kinds or classes". 27 According to this interpretation, what one means by saying that a kind of system has a certain probability to change or to remain in a specific state is that it has a disposition to produce specific long-run frequencies. Here the question emerges to what kind of kind of systems propensities should be attributed. Millstein argues that for probabilities in evolutionary theory a kind is specified by the causal factors that influence population level processes, ignoring details particular to one population such as the relative locations of organisms within the environment. For our purposes it is important that since this interpretation attributes a propensity to a kind of system, the probabilities are in principle dispensable for predictive purposes. Besides, according to this interpretation, the probabilities are ontic because they correspond to features of kinds of systems.

Like all the major interpretations of probability, propensity interpretations are controversial. $^{28}$ The main concerns are to explain what exactly a propensity is, and whether one can accept that a propensity, which is a very peculiar sort of entity, type of causation or property, is a part of the world. These problems are serious. Yet, in my opinion, they do not imply that the propensity interpretation is doomed to failure but rather call for further clarification or research. For Sober the main problem of the propensity interpretation in evolutionary theory is Humphrey's paradox, viz. that the propensity interpretation cannot make sense of backward probabilities as they appear, for example, in coalescence theory. ${ }^{29}$ I will now argue that these backward probabilities do not present a problem.

Coalescence theory gives probabilities of how long ago the most recent ancestor of two organisms existed. A simple model of coalescence theory is as follows: the population number is constant, i.e., there are $N$ organisms in each generation; the likelihood that an organism is a parent of an organism in the next generation is $1 / N$; and the parents of the organisms in a generation are probabilistically independent. Under these assumptions, the probability that the first two organisms of a generation share a parent is $1 / \mathrm{N}$, and the probability that the most recent common ancestor existed $t$ generations in the past is $(1-1 / N)^{t-1}(1 / N)$. These probabilities are backward probabilities in the sense that the question is whether for two organisms which live now the most recent common ancestor existed $t$ generations in the past. For such backward probabilities the worry is that there are no nontrivial propensities: the linage of the two organisms is determined. Hence the two organisms either have or do not have the most recent common ancestor $t$ generations in the past.

For single-case propensity interpretations such as Giere's interpretation discussed above, this might present a problem. However, there is no problem for the propensity interpretation in evolutionary theory, which appeals to kinds of systems. For the simple model of coalescence theory, consider the kind of system where there are $N$ organisms at the start and the organisms reproduce over $t$ generations. Then the probability that the first two organisms have the most recent ancestor $t$ generations in the past is the

\footnotetext{
${ }^{27}$ Millstein "Interpretations of Probability in Evolutionary Theory", loc. cit., p.1324, original emphasis.

${ }^{28}$ Colin Howson and Peter Urbach, Scientific Reasoning, the Bayesian Approach. Peru/Illinois: Open Court 1996, pp.338-351; Mellor "Probability: A Philosophical Introduction", loc. cit., Section 4.

${ }^{29}$ Sober "Evolutionary Theory and the Reality of Macro Probabilities", loc. cit.
} 
propensity of this kind of system to produce a first and a second organism in the $t$-th generation which have their most recent common ancestor $t$ generations in the past. Thus, there is nothing like a backward propensity here. For each run of the system the first two organisms either have or to not have their most recent ancestor $t$ generations in the past. Yet this is entirely compatible with a nontrivial propensity of a system to produce organisms that have their most common ancestor $t$ generations in the past. ${ }^{30}$ To conclude, backward probabilities do not represent a problem for propensity theories in evolutionary theory, which appeal to kinds of systems.

\subsection{Frequency Interpretation}

The second interpretation is the frequency interpretation. According to the most widely accepted version, the probability is the frequency of a hypothetical infinite sequence of trials. In our context it is important to note that, according to the frequency interpretation, probabilities are ontic because the frequencies correspond to real features of the world. Furthermore, because the notion of a frequency applies to sequences of outcomes, the probabilities are in principle dispensable for predictive purposes.

Frequentists are confronted with difficult questions. ${ }^{31}$ A serious worry is that the frequency interpretation overstates the relation of probabilities to frequencies. As treated in the mathematical field of probability theory, a probability can also lead to an infinite sequence of outcomes where the frequency of the sequence differs from the probability. For instance, a fair coin can land heads each time in an infinite run of tosses (though this sequence has probability zero). It is plausible to demand that interpretations of probability should allow for this too, but the frequency interpretation does not. ${ }^{32}$ There is no way out of this by postulating that the probability for an infinite sequence to yield the correct frequency is one. Clearly, this would be circular because probability would be defined by referring to probability. Another problem for hypothetical limiting frequentists is to explain what exactly fixes the outcomes of hypothetical infinite sequences, why counterfactual frequencies are determinate, and why they agree with the probability. ${ }^{33}$ Furthermore, what can happen more or less frequently is not that a single experiment yields an outcome but that members of some class of experiments yield an outcome. This class is called a reference class, and frequentists have to answer the question of what constitutes a reference class. That this problem is difficult is illustrated by the fact that it is easy to change the order of an infinite sequence such that the frequency changes. Thus an answer to the reference class problem also needs to explain why only a certain order of experiments is allowed and others are not allowed.

\footnotetext{
${ }^{30}$ This solution to Humphrey's paradox in evolutionary theory is similar to the solution proposed by Gillies and Mc Curdy. See Donald Gillies, "Varieties of Propensities", in: The British Journal for the Philosophy of Science 51, 4, 2000, pp.807-835; Christoper S. I. McCurdy, "Humphreys's Paradox and the Interpretation of Inverse Conditional Propensities", in: Synthese 108, 1, 1996, pp.105-125.

31 Howson and Urbach "Scientific Reasoning, the Bayesian Approach", loc. cit. 319-337; Mellor "Probability: A Philosophical Introduction", loc. cit., Section 3.

${ }^{32}$ Sober "Evolutionary Theory and the Reality of Macro Probabilities", loc. cit.

${ }^{33}$ Marshall Abrams, "Infinite Populations and Counterfactual Frequencies in Evolutionary Theory", in: Studies in History and Philosophy of the Biological and Biomedical Sciences 37, 2, 2006, pp.256-268; Mellor "Probability: A Philosophical Introduction", loc. cit., Section 3.
} 
In conclusion, the frequency interpretation faces serious problems. In my opinion, they do not imply that the frequency interpretation is doomed to failure. Yet, some of the problems seem hard to solve, and further work is needed to make progress on these problems. Millstein ${ }^{34}$ has argued that the frequency interpretation is of no use in evolutionary theory because it faces an insurmountable problem involving the change of frequencies. I will now argue that Millstein's objection is misguided.

Millstein's argument starts from considering random drift - a process where physical differences between organisms are causally irrelevant to differences in reproductive success. A simple model of drift is as follows: ${ }^{35}$ suppose that the population size is a constant $N$ with $2 N$ alleles and that there are $i$ alleles of type $A$. Further, suppose that the number of alleles of type $A$ in the next generation is the sum of $2 N$ independent Bernoulli variables where the probability for an allele of type $A$ is $i / 2 N$ (the ratio of allele $A$ in the current population). Then the probability that the population will go from $i$ alleles of type $A$ to $j$ alleles of type $A$ is: ${ }^{36}$

$$
p_{i j}=\frac{2 N !}{(2 N-j) ! j !}\left(\frac{i}{2 N}\right)^{j}\left(1-\frac{i}{2 N}\right)^{2 N-j} \text {. }
$$

Clearly, this implies that when drift occurs over a number of generations, the ratio of alleles of type $A$ can fluctuate from generation to generation, especially in small populations. Any interpretation of probability in evolutionary theory has to be able to successfully interpret these probabilities. Millstein argues that these probabilities cannot be interpreted as frequencies because "frequencies may increase, decrease, or remain constant. In an ensemble of populations, eventually each population undergoing drift will go to fixation for one of the types, but which type cannot be predicted". ${ }^{37}$

However, Millstein's worries are unjustified. All the frequency interpretation says for the simple model of drift is that if, again and again, one considers a population with $2 N$ alleles and $i$ alleles of type $A$, the frequency that such a population will go to $j$ alleles of type $A$ is $p_{i j}$. This is entirely consistent with the fact that the ratio of alleles of type $A$ and the transition probabilities can change from one generation to the next and that populations will go to fixation for one of the types. The point is that for a given reference class the frequencies and hence the probabilities are well defined. If the number of alleles of type $A$ changes in one generation from $i$ to $k(i \neq k)$, then also the probabilities $p_{i j}$ and $p_{k j}$ will be different. However, far from being a problem, this is as it should be because $p_{i j}$ and $p_{k j}$ are the probabilities corresponding to different reference classes.

\subsection{Humean Chances}

As a third interpretation I want to suggest Humean chances as recently endorsed by Frigg and Hoefer as a new interpretation of probabilities in evolutionary theory. ${ }^{38}$ The

\footnotetext{
${ }^{34}$ Millstein "Interpretations of Probability in Evolutionary Theory", loc. cit., p.1322.

${ }^{35}$ Jonathan Roughgarden, Theory of Population Genetics and Evolutionary Ecology: An Introduction. Upper Saddle River: Prentice Hall 1996, pp.65-66.

${ }^{36}$ This equation is a correction of Millstein's equation, where there is a typo.

${ }^{37}$ Millstein "Interpretations of Probability in Evolutionary Theory", loc. cit., p.1322.

${ }^{38}$ Roman Frigg and Carl Hoefer, "Determinism and Chance from a Humean Perspective", in: Dennis Dieks, Wenceslao Gonzalez, Stephan Hartmann, Marcel Weber, Friedrich Stadler and Thomas Uebel (Eds.), The Present Situation in the Philosophy of Science. Berlin: Springer 2010, pp.351-372; Carl
} 
Humean mosaic is the collection of all events that actually happen at all times. (Here Frigg and Hoefer make the assumption of ontological pluralism, i.e., entities at different levels of the world, and not only the entities at the most fundamental level, are real.) Humean chances supervene on the Humean mosaic. More specifically, imagine all possible systems of probability rules about events in the Humean mosaic. There will be a best system in the sense that the probability rules of this system can best account for the Humean mosaic in terms of simplicity, strength and fit. The strength of a system of rules is measured by its scope to account for large parts of the Humean mosaic, and fit is measured in terms of closeness to actual frequencies. Then Humean chances are the numbers that are assigned to events by the probability rules of this best system. The reason why the best system contains rules about macroprocesses, such as the processes involving the kinds postulated by evolutionary theory, is simplicity in derivation: even if it were the case that the facts about macroprocesses could be derived from fundamental physics, "it is hugely costly to start from first principles every time you want to make a prediction about the behaviour of a roulette wheel. So the system becomes simpler in that sense if we write in rules about macro objects". 39

Proponents of Humean chances are confronted with the difficult question of how to characterise simplicity, strength and fit in detail. Providing a detailed account of simplicity, strength and fit is crucial because otherwise it remains vague and unclear what probabilities really are. For our purposes it is important to note that because Humean chances are facts entailed by actual events in the world, probabilities, thus understood, correspond to real features of the world. Furthermore, Humean chances as described above differ from Lewis's original proposal in that laws and chances are not analysed together, which implies that the interpretation presented here can also apply to probabilities which are in principle dispensable for predictive purposes. ${ }^{40}$ Indeed, Frigg and Hoefer's main concern is to argue for Humean chances as an account of ontic probabilities in deterministic worlds. In particular, they defend Humean chances as an interpretation of probability in statistical mechanics and as an interpretation of the probabilities associated with deterministic processes such as coin tossing and the spinning of roulette wheels.

In sum, propensities of kinds of systems, frequencies and Humean chances are possible interpretations of probabilities in evolutionary theory in the sense that the probabilities are ontic and can in principle be eliminated for predictive purposes.

\section{Criticism of Sansom's Claim that Biological Processes Are Indeterministic}

Because probabilities are ontic in evolutionary theory, Sansom ${ }^{41}$ concludes that biological processes are really indeterministic. This section will argue that Sansom's argument is inconclusive. First of all, Sansom's argument needs to be introduced in more detail. Sansom distinguishes between two kinds of realism, which he regards as

Hoefer, “The Third Way on Objective Probability: A Sceptic's Guide to Objective Chance”, in: Mind 116, 463, 1007, pp.549-596.

${ }^{39}$ Frigg and Hoefer "Determinism and Chance from a Humean Perspective", loc. cit., p.21.

${ }^{40}$ Hoefer "The Third Way on Objective Probability: A Sceptic's Guide to Objective Chance, loc. cit., pp.558-560.

${ }^{41}$ Sansom "Why Evolution is Really Indeterministic, Ibid. 
the only two versions of realism worthy of further consideration: innocent pluralism and monorealism. Innocent pluralism asserts that different theories describing the same part of the world at different levels can be true and that no level of the world is privileged. ${ }^{42}$ On this view, for instance, the same part of the world can be adequately described by quantum theory and macrophysics. Monorealism holds that the world is truly described by only one theory. For example, some physicists and philosophers have contended that quantum theory is the only theory capturing reality.

Imagine an innocent pluralist who thinks that quantum theory and macrophysics truly describe the world watching a ball rolling across a table. Then, assuming that macrophysics is deterministic and that quantum theory is indeterministic, from the innocent pluralist's point of view the process is indeterministic relative to quantum theory and deterministic relative to macrophysics. Consequently, as Sansom correctly remarks, an innocent pluralist has to accept the "relativity of determinism", namely that the world is neither merely deterministic nor indeterministic, but that whether or not determinism is true is relative to the kinds under consideration.

Sansom argues for realism about evolutionary theory and innocent pluralism by referring to Geach's ${ }^{43}$ view of relative identity. Because processes are indeterministic relative to the kinds posited by evolutionary theory, Sansom concludes that biological processes are really indeterministic.

Sansom is right that processes are indeterministic relative to the kinds posited by evolutionary theory. However, the question arises why one should exclusively focus on the kinds posited by evolutionary theory. To understand this point, a comparison with physics will help. For an innocent pluralist there are many physical realities - the processes relative to quantum-mechanical kinds, the processes relative to the kinds posited by general relativity theory, the processes relative to statistical-mechanical kinds etc. Now suppose that in biology there are also two realities: processes involving life relative to the kinds posited by evolutionary theory and processes involving life relative to macro-physical kinds. Relative to the macro-physical kinds the processes might be deterministic. Then the question whether biological processes are deterministic has no clear answer for an innocent pluralist: biological processes are indeterministic relative to the kinds posited by evolutionary theory and deterministic relative to the macro-physical kinds.

Sansom's concern are the biological realities as considered by biologists and philosophers of biology. ${ }^{44} \mathrm{He}$ simply assumes and does not provide any argument for the exclusive focus on the biological reality of the processes relative to the kinds posited by evolutionary theory. Is there no need to justify this assumption because it is uncontroversial that there is only one biological reality, viz. the processes involving life relative to the kinds posited by evolutionary theory? This is not so. The extant literature speaks at least about two biological realities: namely, about a biological reality of the processes involving life relative to the kinds posited by evolutionary theory, and about another biological reality of the processes involving life relative to

\footnotetext{
${ }^{42}$ Sansom introduces this concept by alluding to the presentation of this view by Sober - see Elliott Sober, The Nature of Selection. Cambridge/MA: MIT Press 1984.

${ }^{43}$ Peter Geach (1973), "Ontological Relativity and Relative Identity”, in: Milton K. Munitz (Ed.), Logic and Ontology. New York: New York University Press 1973, pp.287-302.

${ }^{44}$ Clearly, Sansom cannot arbitrarily decide what to call "biological reality" because this would render his argument uninteresting.
} 
macro-physical kinds. Important for our purpose is that the latter is standardly referred to as a biological reality. ${ }^{45}$ Indeed, there is a lively debate in the philosophy of biology about the question whether determinism holds true for the biological reality of the processes involving life relative to macro-physical kinds. As already mentioned in Section 2, Rosenberg ${ }^{46}$ argues that this biological reality is deterministic. Abrahams ${ }^{47}$ and Graves et al. ${ }^{48}$ claim that it is indeterministic but that all probabilities are very close to zero and one. Others such as Millstein ${ }^{49}$ and Weber ${ }^{50}$ argue that we do not know enough about the role of quantum events at the macroscopic level and hence should remain agnostic about whether or not this biological reality is deterministic.

To conclude, Sansom simply assumes that "biological reality" refers to the processes relative to the kinds posited by evolutionary theory, but this assumption is not justified. The extant literature speaks at least about two biological realities - processes involving life relative to the kinds posited by evolutionary theory and processes involving life relative to macro-physical kinds. Consequently, for an innocent pluralist the question whether biological processes are deterministic has to broken up into (at least) two subquestions: Are processes involving life deterministic relative to the kinds posited by evolutionary theory? Are processes involving life deterministic relative to macro-physical kinds? Hence for Sansom's argument to be tenable, he would need to show that biological processes are indeterministic relative to these two sets of kinds. However, he has not shown that processes involving life are indeterministic relative to macro-physical kinds. And, as illustrated by the debate in philosophy of biology ${ }^{51}$, the question whether biological processes are deterministic relative to macro-physical kinds is controversial and has no easy answer. Consequently, Sansom's argument that biological processes are really indeterministic (for an innocent pluralist) does not succeed.

\section{Conclusion}

Probability and indeterminism have always been central philosophical themes. This paper contributed to understanding these themes by investigating probability and indeterminism in biology.

The starting point was the following argument: an omniscient being would not need the probabilities of evolutionary theory to make predictions. Despite this, one can still

\footnotetext{
${ }^{45}$ Abrams, "Fitness and Propensity's Annulment?" loc. cit.; Millstein "Interpretations of Probability in Evolutionary Theory", loc. cit.; Millstein, "Is the Evolutionary Process Deterministic or Indeterministic?", loc. cit.; Rosenberg "Instrumental Biology or the Disunity of Science” loc. cit.; Rosenberg, "Discussion Note: Indeterminism, Probability, and Randomness in Evolutionary Theory", loc. cit.

${ }^{46}$ Rosenberg "Instrumental Biology or the Disunity of Science” loc. cit.

${ }^{47}$ Abrams, "Fitness and Propensity's Annulment?" loc. cit.

${ }^{48}$ Graves et al. "Is Indeterminism the Source of the Probabilistic Character of Evolutionary Theory?" loc. cit.

${ }^{49}$ Millstein, "Is the Evolutionary Process Deterministic or Indeterministic?", loc. cit.

${ }^{50}$ Weber "Indeterminism in Neurobiology", loc. cit.

${ }^{51}$ Robert N. Brandon and Scott Carson, "The Indeterministic Character of Evolutionary Theory: No 'No Hidden Variables Proof' but Not Room for Determinism Either', in: Philosophy of Science 63, 3, 1996, pp.315-337; Graves et al. "Is Indeterminism the Source of the Probabilistic Character of Evolutionary Theory?" loc. cit.; Millstein, "Is the Evolutionary Process Deterministic or Indeterministic?", loc. cit.
} 
be a realist about evolutionary theory. For a realist about evolutionary theory the probabilities are ontic, i.e., they refer to real features of the world. This prompted the question of how to understand probabilities which are ontic but which are in principle dispensable for predictive purposes.

The contribution of the paper to this question was to suggest three possible interpretations of such probabilities in evolutionary theory. The first interpretation was a propensity interpretation of kinds of systems. Since this interpretation attributes a propensity to kinds of system, the probabilities are ontic and are in principle dispensable for predictive purposes. Sober's objection that propensity theories cannot deal with backward probabilities in biology was discussed. By investigating backward probabilities in coalescence theory, I concluded that backward probabilities are unproblematic because they can be understood as propensities of kinds of systems. The second interpretation was the frequency interpretation. Since a frequency applies to a sequence of outcomes, the probabilities are ontic and are in principle dispensable for predictive purposes. I examined Millstein's objection that in the case of drift frequencies often change, implying that biological probabilities cannot be interpreted as frequencies. I argued that this objection is beside the point because it is normal that there are different frequencies for different reference classes. Third, I suggested Humean chances as a new interpretation of probability in biology. Humean chances are the numbers assigned to events by the probabilities rules of the best system (the best system is identified by the probability rules that can best account for the collection of all actual events in terms of simplicity, strength and closeness to frequencies). Humean chances are ontic because they are facts entailed by all actual events. Furthermore, because of simplicity of derivation, probabilities are also assigned to macro-processes, and hence Humean chances are in principle dispensable for predictive purposes. All three interpretations suffer from problems, and further research is required to tackle them. Yet they at least show us three possible ways of understanding ontic probabilities in evolutionary theory.

Finally, I criticised Sansom's claim that biological processes are really indeterministic. Sansom is a realist about evolutionary theory and subscribes to the view that different theories describing the same part of the world at different levels can be true. Because processes are indeterministic relative to the kinds posited by evolutionary theory, Sansom concludes that biological processes are indeterministic. Sansom's argument presupposes that "biological reality" refers to the processes relative to the kinds posited by evolutionary theory. However, this assumption is not justified. The extant literature in biology and philosophy is concerned with at least two biological realities - processes involving life relative to the kinds posited by evolutionary theory and processes involving life relative to macro-physical kinds. Consequently, Sansom's argument that evolution is really indeterministic is not conclusive. The problem whether biological processes are deterministic or indeterministic is still with us. 